\title{
MAXIMUM THEOREMS FOR SOLUTIONS OF HIGHER ORDER ELLIPTIC EQUATIONS
}

\author{
BY SHMUEL AGMON \\ Communicated by Lipman Bers, December 4, 1959
}

The classical maximum modulus theorem for solutions of second order elliptic equations was recently extended by C. Miranda [4] to the case of real higher order elliptic equations in two variables. Previously Miranda [3] has derived a maximum theorem for solutions of the biharmonic equation in two variables. In the case of more variables it was observed by Agmon-Douglis-Nirenberg [2] that a maximum theorem holds in the special case of elliptic operators with constant coefficients with no lower order terms when the domain of definition is a half-space.

In this note we describe a very general maximum theorem for solutions of (complex) higher order elliptic equations in any number of variables. We shall obtain various estimates in the maximum norm which will contain as a special case the extension of Miranda's results to any number of variables.

We denote by $G$ a bounded domain in $E_{n}$ with boundary $\partial G$ and closure $\bar{G}$. For a function $u \in C^{i}(\bar{G})$ we introduce the usual maximum norm:

$$
\|u\|_{j}^{\bar{G}}=\max _{|\alpha| \leqq} \max _{x \in \bar{G}}\left|D^{\alpha} u(x)\right| \text {. }
$$

Here $\alpha=\left(\alpha_{1}, \cdots, \alpha_{n}\right)$ is a multiple index of length $|\alpha|=\alpha_{1}+\cdots$ $+\alpha_{n}$ and $D^{\alpha}$ is the corresponding partial derivative. Furthermore, for continuous functions $u$ in $\bar{G}$ we introduce negative maximum norms $\|u\|_{-j}^{G}(j>0)$ defined in the following manner. Write $u$ in the form

$$
u=\sum_{|\alpha| \leqq j} D^{\alpha} f_{\alpha}
$$

with $f_{\alpha} \in C^{|\alpha|}(\bar{G})$. Then:

$$
\|u\|_{-j}^{G}=\operatorname{Inf} \max _{|\alpha| \leqq j}\left\|f_{\alpha}\right\|_{0}^{\bar{G}},
$$

where the infimum is taken over all possible representations of the form (2).

Actually we are going to use negative norms for functions $f$ defined on the (sufficiently smooth) boundary. If $f$ has continuous derivatives 
up to the order $j \geqq 0$ on $\partial G$ then one defines the $j$ th maximum norm $\|f\|_{j}^{\partial G}$ in the usual way by means of local coordinates taking note of (1). Similarly it is obvious from (3) how one defines by means of local coordinates the negative norm $\|f\|_{-j}^{\partial G}(j>0$ and $f$ is continuous). Finally, for $u \in C^{j}(G)$ we also define the following $L_{p}$ norm:

Now let

$$
\|u\|_{j, L_{p}(G)}=\left(\int \sum_{G|\alpha| \leqq j}\left|D^{\alpha} u\right|^{p} d x\right)^{1 / p} .
$$

$$
A=\sum_{|\alpha| \leqq 2 m} a_{\alpha}(x) D^{\alpha}
$$

be â (complex) elliptic operator of order $2 m$ in $\bar{G}$. Suppose that $a_{\alpha} \in C^{|\alpha|}(\bar{G}), G$ being of class $C^{2 m}$. In the case of two variables we also assume that $A$ satisfies the "roots condition" (see, for instance, [2]) a condition which is always satisfied for real elliptic operators or when the number of variables is at least three. We consider now functions $u \in C^{m-1}(\bar{G}) \cap C^{2 m}(G)$ such that

$$
\begin{array}{ll}
A u=0 & \text { in } G, \\
\frac{\partial^{j} u}{\partial n^{j}}=\phi_{j} & \text { on } \partial G, j=0, \cdots, m-1,
\end{array}
$$

$(\partial / \partial n$ denotes differentiation along the normal). The main result is the following

THEOREM I. Let $l$ be an integer such that $0 \leqq l \leqq m-1$. Then, for all functions $u$ satisfying (6) the following estimate holds:

$$
\|u\|_{l}^{\bar{G}} \leqq c \sum_{j=0}^{m-1}\left\|\phi_{j}\right\|_{l-j}^{\partial G}+c_{1}\|u\|_{L_{1}(G)},
$$

where $c, c_{1}$ are constants depending on $A$ and $G$ but not on $u$. If, moreover, the solution of the Dirichlet problem (6) is unique in a suitable (small) class of functions then (7) holds with $c_{1}=0$.

We note that the extension of Miranda's results corresponds to the case $l=m-1$. If $l<m-1$ then (7) contains negative norms on the right hand side (replacing these norms by the zero norm one obtains a weaker result). In particular, taking $l=0$ and assuming uniqueness, one obtains the estimate:

$$
\max _{\bar{G}}|u| \leqq c \sum_{j=0}^{m-1}\left\|\phi_{j}\right\|_{-j}^{\partial G} \leqq c_{0} \sum_{j=0}^{m-1} \max _{\partial G}\left|\phi_{j}\right| .
$$

Combining known existence results for the Dirichlet problem for 
smooth data with, for instance, the estimate (8), one obtains easily a solution of the Dirichlet problem (6) when the given data $\phi_{j}$ are merely continuous. It is an ordinary solution of the equation in the interior, continuous in $\bar{G}, u=\phi_{0}$ on $\partial G$, while the other Dirichlet data are taken in a generalized sense.

The method of proof of Theorem I uses an artifice introduced by Miranda in [4]. It consists in constructing a good "approximate solution" $u_{0}$ of (6) which takes the same Dirichlet data as $u$. For this purpose the Poisson kernels which resolve explicitly the Dirichlet problem for elliptic operators with constant coefficients in a halfspace are used. These kernels were given in [2]. One then shows that the approximate solution $u_{0}$ satisfies (7) with $c_{1}=0$. Thus the problem is reduced to showing that the function $u_{1}=u-u_{0}$ (which has zero Dirichlet data) satisfies (7). This is done with the aid of the following $L_{p}$ estimates for elliptic operators established recently by the author [1] (combined with Sobolev's inequalities).

Theorem II. Let $u \in C^{k}(G) \cap L_{q}(G)$ for some $q>1$. Let $p>1$, $p^{\prime}=p /(p-1)$. Suppose that for all functions $v \in C^{2 m}(\bar{G})$ such that $\partial^{i} v / \partial n^{j}=0$ on $\partial G(0 \leqq j \leqq m-1)$ the following inequality holds:

$$
\left|\int_{G} u \bar{A} \bar{v} d x\right| \leqq C_{u}\|v\|_{2 m-k, L_{p^{\prime}}(G)},
$$

where $C_{u}$ is some constant depending only on $u$. Then:

$$
\|u\|_{k, L_{p}(\theta)} \leqq c_{0} C_{u}+c_{1}\|u\|_{L_{1}(G)}
$$

where $c_{0}, c_{1}$ are constants depending on the elliptic operator $A$ and the domain but not on $u$. If, moreover, the solution of the Dirichlet problem (6) is unique then (10) holds with $c_{1}=0$.

We shall illustrate the method of proof of Theorem I (in particular the manner in which Theorem II is used) in a special case where the construction of a good approximate solution $u_{0}$ is particularly simple. Consider a fourth order elliptic operator in the plane of the form:

$$
A=\Delta^{2}+A_{1}
$$

where $A_{1}$ is a lower order operator with variable coefficients. Take $G$ to be a simply connected domain with sufficiently smooth boundary. Since by a conformal mapping the form of $A$ remains unchanged (after division by some factor), we can assume without loss of generality that $G$ is the unit-circle. As a suitable approximate solution one can choose here the solution $u_{0}$ of the biharmonic equation $\Delta^{2} u_{0}=0$ 
which takes the same Dirichlet data as $u$. This solution could be written down explicitly and it is easily verified by inspection that

$$
\left\|u_{0}\right\|_{l}^{\bar{G}} \leqq K\left(\left\|\phi_{0}\right\|_{l}^{\partial G}+\left\|\phi_{1}\right\|_{l-1}^{\partial G}\right), \quad l=0,1,
$$

where $K$ is some absolute constant. Put $u_{1}=u-u_{0}$. We shall now use Theorem II to show that $u_{1}$ satisfies (7). Let $A^{*}$ be the formal adjoint of $A$. By Green's formula it is readily seen that for all functions $v \in C^{4}(\bar{G})$ such that $v=0, \partial v / \partial n=0$ on $\partial G$ :

$$
\int_{G} u_{1} \bar{A}^{*} \bar{v} d x=-\int_{G} A_{1} u_{0} \cdot \bar{v} d x
$$

Integrating the right hand side by parts and using Hölder's inequality we find readily for $l=0,1$ that

$$
\left|\int_{G} u_{1} \bar{A}^{*} \bar{v} d x\right| \leqq c_{2}\left\|u_{0}\right\|_{l, L_{p}(G)}\|v\|_{3-l, L_{p^{\prime}}(G)} \leqq c_{3}\left\|u_{0}\right\|\|\|_{l}^{\bar{G}}\|v\|_{3-l, L_{p^{\prime}}(G)} .
$$

Applying Theorem II to $u_{1}$ (with $m=2, k=l+1$ ), we find that

$$
\left\|u_{1}\right\|_{l+1, L_{p}(G)} \leqq c_{4}\left\|u_{0}\right\|_{l}^{\bar{G}}+c_{5}\left\|u_{1}\right\|_{L_{1}(G)},
$$

with $c_{5}=0$ if uniqueness holds. Choosing now $p>2$ we have by Sobolev's inequalities:

$$
\left\|u_{1}\right\|_{l}^{\bar{G}} \leqq K_{1}\left\|u_{1}\right\|_{l+1, L_{p}(G)} \quad\left(K_{1} \text { constant }\right) .
$$

Combining (15), (14) and (12) we get Theorem I in the special case considered.

\section{REFERENCES}

1. S. Agmon, The $L_{p}$ approach to the Dirichlet problem, Hebrew University, Technical Note no. 7, 1959.

2. S. Agmon, A. Douglis and L. Nirenberg, Estimates near the boundary for solutions of elliptic partial differential equations satisfying general boundary conditions, I, Comm. Pure Appl. Math. vol. 12 (1959).

3. C. Miranda, Formule di maggiorazione e teorema di esistenza per le funzioni biarmoniche di due variabili, Giorn. Mat. Battaglini vol. 78 (1948-1949) pp. 97-118.

4. - - Teorema del massimo modulo e teorema di esistenza e di unicita per il problema di Dirichlet relativo alle equazioni ellittiche in due variabili, Ann. Mat. Pura Appl. vol. 46 (1958) pp. 265-311.

HeBreW UNIVERSITY, JERUSALEM, ISRAEL AND

Institute for Fluid Dynamics and Applied Mathematics, UNIVERSITY OF MARYLAND 